\title{
Spectral sequences and taut Riemannian foliations *
}

\author{
Vladimir Slesar
}

$29 / 04 / 2006$

\begin{abstract}
Using a relation between the terms of the spectral sequence of a Riemannian foliation and its adiabatic limit, we obtain Bochner type techniques for this special setting and, as a consequence, in the special case of a Riemannian flow we obtain vanishing conditions for the top dimensional group of the basic cohomology $H_{b}^{q}(\mathcal{F})$-which is related to the property of being geodesible. We also extend a Weitzenböck type formula for the leafwise Laplacian and, for the particular class of compact foliations, we obtain a generalization of a result due to $\mathrm{Ph}$. Tondeur, M. Min-Oo and E. Ruh concerning the vanishing of the basic cohomology under the assumption that certain curvature operators are positive definite. In the final part we present an example.
\end{abstract}

Keywords: Riemannian foliation; geodesic flow; spectral sequences MSC Classification: 53C12, 53D25, 55T05.

\section{Introduction}

Throughout this paper we consider a Riemannian foliation $\mathcal{F}$ defined on a closed Riemannian manifold $M$ with a bundle-like metric $g$ [Re].

Our paper basically uses the joint works of J. A. Álvarez López and Y. Kordyukov [AlKo1] and [AlKo2]. In these papers the authors introduce a

*Partially supported by Grant Nr. MTM2004-08214 
Hodge-de Rham theory for the spectral sequences of a Riemannian foliation (which is a generalization of the basic Hodge-de Rham theory).

We will start out by considering the $C^{\infty}$ foliation $\mathcal{F}$ on a closed manifold $M$; the dimension of foliation will be denoted by $p$, the codimension by $q$ and $(\Omega, d)$ will denote the de Rham complex on $M$. Let us now consider the following bigrading for $\Omega$, induced by the foliated structure and the bundlelike metric:

$$
\Omega^{u, v}=C^{\infty}\left(\bigwedge^{u} T \mathcal{F}^{\perp *} \oplus \bigwedge^{v} T \mathcal{F}^{*}\right), u, v \in \mathbf{Z} .
$$

Then, the de Rham derivative and coderivative split into bihomogeneous components as follows:

$$
d=d_{0,1}+d_{1,0}+d_{2,-1}, \quad \delta=\delta_{0,-1}+\delta_{1,0}+\delta_{-2,1},
$$

where the indices describe the corresponding bigrading. We notice that $d_{2,-1}$ and $\delta_{-2,1}$ are of 0 -th order and vanish if and only if the distribution $T \mathcal{F}^{\perp}$ is completely integrable.

The spectral sequence $\left(E_{k}, d_{k}\right)$ on $\mathcal{F}$ is defined using the following decreasing filtration:

$$
\Omega=\Omega_{0} \supset \Omega_{1} \supset \ldots \supset \Omega_{q} \supset \Omega_{q+1}=0,
$$

where the space of $r$-forms of filtration degree $\geq k$ is given by:

$$
\Omega_{k}^{r}=\left\{\omega \in \Omega^{r} \mid i_{X} \omega=0,(\forall) X=X_{1} \wedge \ldots \wedge X_{r-k+1}, X_{i} \in \mathfrak{X}(\mathcal{F})\right\} .
$$

In the following, we consider the terms of the differential spectral sequence $\left(E_{k}, d_{k}\right)$ defined in the classical way (see e.g $[\mathrm{McC}]$ ).

The $C^{\infty}$ topology on $\Omega$ induces a topology on each $E_{k}^{u, v}$, with respect to the bigrading. In this manner, each $d_{k}$ becomes a continuous operator on $E_{k}=\bigoplus_{u, v} E_{k}^{u, v}$. So we obtain two bigraded complexes: $\overline{0}_{k} \subset E_{k}$ and the quotient complex $\widehat{E}_{k}=E_{k} / \overline{0}_{k}$.

Considering the sequence of canonically induced operators $d_{k}: E_{k}^{u, v} \rightarrow$ $E_{k}^{u+k, v-k+1}$, J. A. Álvarez López and Y. Kordyukov inductively define a sequence of second order operators of Hodge-de Rham type: $\Delta_{0}, \Delta_{1}, \ldots$ and a corresponding sequence of eigenspaces $\mathcal{H}_{1} \supseteq \mathcal{H}_{2} \supseteq \ldots \supseteq \mathcal{H}_{\infty}$ such that:

$$
\Omega=\mathcal{H}_{1} \oplus \overline{\operatorname{im} d_{0}} \oplus \overline{\operatorname{ker} \delta_{0}},
$$




$$
\mathcal{H}_{1}=\mathcal{H}_{2} \oplus \operatorname{im} d_{1} \oplus \operatorname{ker} \delta_{1}
$$

yielding $\mathcal{H}_{1} \simeq \widehat{E}_{1}, \mathcal{H}_{k} \simeq E_{k}$, for $k=2,3, \ldots \infty$, the above splitting being just the corresponding Hodge-de Rham decomposition.

Now the metric $g$ can be written as $g=g_{\perp} \oplus g_{\mathcal{F}}$ with respect to the decomposition $T M=T \mathcal{F}^{\perp} \oplus T \mathcal{F}$. Introducing a parameter $h>0$, we define the family of metrics

$$
g_{h}=h^{-2} g_{\perp} \oplus g_{\mathcal{F}} .
$$

The limit of the Riemannian manifold $\left(M, g_{h}\right)$ as $h \downarrow 0$ is known as the adiabatic limit. It was introduced by $\mathrm{E}$. Witten for a Riemannian bundle over the circle $[\mathrm{W}]$. In our paper the adiabatic limit procedure will be just a tool in order to achieve Weitzenböck type formula for the terms of the differentiable spectral sequence, this appearing as a natural consequence of the above mentioned Hodge-de Rham theory.

The bundle-like metric induces also a splitting of the cotangent bundle $T M^{*}=T \mathcal{F}^{\perp *} \oplus T \mathcal{F}^{*}$. The canonical transversal and leafwise projection operator will be denoted by $\mathrm{pr}^{\mathcal{T}}$ and $\mathrm{pr}^{\mathcal{L}}$ respectively. We can consider the rescaling homomorphism $\Theta_{h}:\left(T M^{*}, g_{h}\right) \rightarrow\left(T M^{*}, g\right)$, defined using the identity operators $\operatorname{id}_{T \mathcal{F}^{\perp *}}$ and $\operatorname{id}_{T \mathcal{F}^{*}}$ :

$$
\Theta_{h}=h \operatorname{id}_{T \mathcal{F}^{\perp *}} \oplus \mathrm{id}_{T \mathcal{F}^{*}} .
$$

The induced rescaling homomorphism on differential forms or tensor fields will be denoted also by $\Theta_{h}$. One can prove that these are in fact isometries of Riemannian vector bundles (see e.g [MazMe]). The rescaled Laplace operator $\Theta_{h} \Delta_{g_{h}} \Theta_{h}^{-1}$ induces a rescaled Weitzenböck formula (see (22)):

$$
\left\langle\Delta_{h} \omega, \omega\right\rangle=\left\langle\nabla^{h} \omega, \nabla^{h} \omega\right\rangle+\left\langle K^{h} \omega, \omega\right\rangle .
$$

for any $\omega \in \Omega$; the inner product is obtained integrating on the closed Riemannian manifold $M$ (see e.g. [CrPuRa]).

The main ingredient in our further considerations is Proposition 1 which is a direct consequence of Corollary $\mathrm{C}$ in [AlKo2]. This result, together with Theorem B in [AlKo1] offers us a description of a differential form which lies in $\mathcal{H}_{2}^{u, v}$ : 
Proposition 1 For any $\omega^{u, v} \in \Omega^{u, v}$, with $u+v=r$, we have $\omega^{u, v} \in \mathcal{H}_{2}^{u, v}$ if and only if there is a family of differential forms $\omega_{h} \in \Omega^{r}$ depending on $h>0$, so that $\omega_{h} \rightarrow \omega^{u, v}$ in $L^{2}$ norm as $h \downarrow 0$, and

$$
\left\langle\Delta_{h} \omega_{h}, \omega_{h}\right\rangle \in o\left(h^{2}\right) .
$$

Using Proposition 1 and introducing a Bochner type technique for the spectral sequence, in the last section of the paper we prove the following vanishing results for the $E_{2}$ term of the spectral sequence; these results involve the operator $K$ (see (13)). The upper index comes from the polynomial description of $K^{h}$, while the lower indices come from the bigrading (see (24)):

Theorem 1 Considering a Riemannian foliation $(M, \mathcal{F}, g)$, if the differential operators of 0 -th order $K^{0}, K^{1}$ are non-negative operators, and $K^{2}$ is strictly positive, then the second term $E_{2}$ of the spectral sequence vanishes.

Let us consider another curvature function specific to a Riemannian foliation: the mixed scalar curvature (see e.g. [Rov]). At an arbitrary point $x \in M$, we define scal mixed $:=\sum_{i=1}^{p} \sum_{j=1}^{q} R\left(e_{i}, f_{a}, f_{a}, e_{i}\right)$, where $1 \leq i \leq p$ and $1 \leq a \leq q$ such that $f_{a} \in T \mathcal{F}^{\perp}$ and $e_{i} \in T \mathcal{F}$ form an orthonormal basis $\left\{f_{a}, e_{i}\right\}$ at $x$.

We state now the following vanishing result which uses the mixed scalar curvature associated to the Riemannian type tensor $R^{0}$ (see (10)):

Theorem 2 If $(M, \mathcal{F}, g)$ is a Riemannian foliation with scal ${ }_{\text {mixed }}^{0}>0$ on $M$, the tangent Ricci operator $R i c^{\mathcal{F}}$ is non-negative and furthermore the operator $K_{-1,1}^{1}$ is non-negative, then $H_{b}^{q}(\mathcal{F})=0$.

An interesting result is related to the case $p=1$-the so called Riemannian flows:

Corollary 1 If $(M, \mathcal{F}, g)$ is a Riemannian flow so that $s c a l_{\text {mixed }}^{0}>0$ on $M$, then $H_{b}^{q}(\mathcal{F})=0$, and the foliation is not "geodesible".

In dimension 3, the topological aspects of Riemannian flows were classified by Y. Carrière in [Car]. In higher dimension aspects related to the basic cohomology, more exactly vanishing results involving basic cohomology groups might be obtained using the above techniques. 
In the last part of this paper we apply the above considerations for a $\mathrm{SO}(3, \mathbf{R})$-foliation in order to obtain concrete vanishing results.

The results contained in this paper are part of my $\mathrm{PhD}$ Thesis [Sl] and were presented within the Conference Foliations 2005, held in Lodz, Poland.

Finally, I would like to thank Jesús A. Álvarez López and Y. Kordyukov for helpful comments made about this paper. Also, I would like to thank the referee for observations and suggestions.

\section{Levi-Civita connection and adiabatic limits}

In what follows let us consider $\left\{F_{a}\right\}, 1 \leq a \leq q$, as being $C^{\infty}$ local infinitesimal transformation of $(M, \mathcal{F})$ orthogonal to the leaves, while $\left\{E_{i}\right\}$, $1 \leq i \leq p$, will be $C^{\infty}$ local vector fields tangent to the leaves. Furthermore, assume that $\left\{F_{a}, E_{i}\right\}$ determine an orthonormal basis $\left\{f_{a}, e_{i}\right\}$ at any point where they are defined. Let us consider also the dual coframes $\left\{\theta^{a}, \omega^{i}\right\}$ for $\left\{F_{a}, E_{i}\right\}$, and $\left\{\alpha^{a}, \beta^{i}\right\}$ for $\left\{f_{a}, e_{i}\right\}$. We denote by $U^{\mathcal{T}}$ the transverse component and by $U^{\mathcal{L}}$ the leafwise component of a local tangent vector field $U$. For the sake of simplicity, the local vector fields $\left\{F_{a}\right\}$ will be called basic vector fields (see e.g $[\mathrm{Mo}]$ ). In the following we consider arbitrary local tangent vector fields $U, V, Z$ and $W$. First of all, let us remind the Gray-O'Neill tensors fields $A$ and $T[\mathrm{ON}]$ :

$$
\begin{aligned}
& T_{U} V:=\nabla_{U^{\mathcal{L}}}^{\mathcal{L}} V^{\mathcal{T}}+\nabla_{U^{\mathcal{L}}}^{\mathcal{T}} V^{\mathcal{L}}, \\
& A_{U} V:=\nabla_{U^{\mathcal{T}}}^{\mathcal{L}} V^{\mathcal{T}}+\nabla_{U^{\mathcal{T}}}^{\mathcal{T}} V^{\mathcal{L}},
\end{aligned}
$$

where $\nabla$ is the Levi-Civita connection. Using the classical Koszul formula

$$
\begin{aligned}
2 g\left(\nabla_{U} V, Z\right)= & U(g(V, Z))+V(g(U, Z))-Z(g(U, V)) \\
& +g([U, V], Z)+g([Z, U], V)+g(U,[Z, V]),
\end{aligned}
$$

and considering the similar formula for the metric $g_{h}$, we are able to express all the components of the Levi-Civita connection (determined by the transverse-tangent decomposition) as polynomials in $h$. We obtain:

Proposition 2 The canonical Levi-Civita connections associated to the metrics $g_{h}$ and $g$ are related by the following relations: 


$$
\begin{array}{ll}
\nabla_{F_{a}}^{g_{h}, \mathcal{T}} F_{b}=\nabla_{F_{a}}^{\mathcal{T}} F_{b}, & \nabla_{F_{a}}^{g_{h}, \mathcal{L}} F_{b}=\nabla_{F_{a}}^{\mathcal{L}} F_{b}=A_{F_{a}} F_{b}, \\
\nabla_{E_{h}}^{g_{h}}, \mathcal{T} E_{j}=h^{2} \nabla_{E_{i}}^{\mathcal{T}} E_{j}=h^{2} T_{E_{i}} E_{j}, & \nabla_{E_{h}}^{g_{h}, \mathcal{L}} E_{j}=\nabla_{E_{i}}^{\mathcal{L}} E_{j}, \\
\nabla_{F_{a}}^{g_{h}} E_{i}=h^{2} \nabla_{F_{a}}^{\mathcal{T}} E_{i}=h^{2} A_{F_{a}} E_{i}, & \nabla_{F_{a}}^{g_{h}, \mathcal{L}} E_{i}=\nabla_{F_{a}}^{\mathcal{L}} E_{i}, \\
\nabla_{E_{i}}^{g_{h}, \mathcal{T}} F_{a}=h^{2} \nabla_{E_{i}}^{\mathcal{T}} F_{a}=-h^{2} A_{F_{a}}^{*} E_{i}, & \nabla_{E_{i}}^{g_{h}, \mathcal{L}} F_{a}=\nabla_{E_{i}}^{\mathcal{L}} F_{a}=T_{E_{i}} F_{a},
\end{array}
$$

for arbitrary indices $a, b, c, i, j$ and $k$, with $1 \leq a, b, c \leq q$ and $1 \leq i, j, k \leq p$, respectively.

Proof. In our setting $g\left(E_{i}, E_{j}\right)$ and $g\left(F_{a}, F_{b}\right)$ are constant functions, $g\left(V^{\mathcal{T}}, W^{\mathcal{L}}\right)=0$, and we have similar relations for the metric $g_{h}$. Also $\left[E_{i}, F_{a}\right]^{\mathcal{T}}=0, F_{a}$ being basic local vector field. As a consequence, using Koszul formula, we get:

$$
\begin{aligned}
2 g_{h}\left(\nabla_{F_{a}}^{g_{h}} F_{b}, F_{c}\right) & =g_{h}\left(\left[F_{a}, F_{b}\right], F_{c}\right)+g_{h}\left(\left[F_{c}, F_{a}\right], F_{b}\right)+g_{h}\left(F_{a},\left[F_{c}, F_{b}\right]\right) \\
& =h^{-2}\left(g\left(\left[F_{a}, F_{b}\right], F_{c}\right)+g\left(\left[F_{c}, F_{a}\right], F_{b}\right)+g\left(F_{a},\left[F_{c}, F_{b}\right]\right)\right) \\
& =2 h^{-2} g\left(\nabla_{F_{a}} F_{b}, F_{c}\right), \\
2 g_{h}\left(\nabla_{F_{a}}^{g_{h}} F_{b}, E_{i}\right) & \left.=g_{h}\left(\left[F_{a}, F_{b}\right], E_{i}\right)=g\left(\left[F_{a}, F_{b}\right], E_{i}\right)\right)=2 g\left(\nabla_{F_{a}} F_{b}, E_{i}\right), \\
2 g_{h}\left(\nabla_{E_{i}}^{g_{h}} E_{j}, F_{a}\right) & =g_{h}\left(\left[F_{a}, E_{i}\right], E_{j}\right)+g_{h}\left(E_{i},\left[F_{a}, E_{j}\right]\right)=2 g\left(\nabla_{E_{i}} E_{j}, F_{a}\right), \\
2 g_{h}\left(\nabla_{E_{i}}^{g_{h}} E_{j}, E_{k}\right) & =g_{h}\left(\left[E_{i}, E_{j}\right], E_{k}\right)+g_{h}\left(\left[E_{k}, E_{i}\right], E_{j}\right)+g_{h}\left(E_{i},\left[E_{k}, E_{j}\right]\right) \\
& =g\left(\left[E_{i}, E_{j}\right], E_{k}\right)+g\left(\left[E_{k}, E_{i}\right], E_{j}\right)+g\left(E_{i},\left[E_{k}, E_{j}\right]\right) \\
& =2 g\left(\nabla_{E_{i}} E_{j}, E_{k}\right) .
\end{aligned}
$$

Now, considering that $g_{h}\left(\nabla_{F_{a}}^{g_{h}} F_{b}, F_{c}\right)=h^{-2} g\left(\nabla_{F_{a}}^{g_{h}} F_{b}, F_{c}\right), g_{h}\left(\nabla_{E_{i}}^{g_{h}} E_{j}, F_{a}\right)=$ $h^{-2} g\left(\nabla_{E_{i}}^{g_{h}} E_{j}, F_{a}\right), g_{h}\left(\nabla_{F_{a}}^{g_{h}} F_{b}, E_{i}\right)=g\left(\nabla_{F_{a}}^{g_{h}} F_{b}, E_{i}\right), g_{h}\left(\nabla_{E_{i}}^{g_{h}} E_{j}, E_{k}\right)=g\left(\nabla_{E_{i}}^{g_{h}} E_{j}, E_{k}\right)$, we obtained the first four relations. The last four relations can be obtained in the following way: as $\left\{F_{a}, E_{i}\right\}$ is orthonormal, the following relations hold

$$
\begin{aligned}
g\left(\nabla_{U} E_{i}, F_{a}\right) & =-g\left(E_{i}, \nabla_{U} F_{a}\right), \\
g_{h}\left(\nabla_{U}^{g_{h}} E_{i}, F_{a}\right) & =-g_{h}\left(E_{i}, \nabla_{U}^{g_{h}} F_{a}\right),
\end{aligned}
$$

for an arbitrary vector field $U$ on $M$ and, as a consequence, we get $\nabla_{F_{a}}^{g_{h}} \mathcal{T} E_{i}=$ $h^{2} \nabla_{F_{a}}^{\mathcal{T}} E_{i}$ and $\nabla_{F_{a}}^{g_{h}, \mathcal{L}} E_{i}=\nabla_{F_{a}}^{\mathcal{L}} E_{i}$. Finally, as the torsion tensor fields $T$ and $T_{g_{h}}$ vanish, we obtain the last two relations. 
Proposition $3 \nabla$ and $\nabla^{g_{h}}$ satisfy

$$
\begin{array}{lll}
\nabla_{F_{a}}^{g_{h}, \mathcal{T}} \theta^{b}=\nabla_{F_{a}}^{\mathcal{T}} \theta^{b}, & \nabla_{F_{a}}^{g_{h}, \mathcal{L}} \theta^{a}=h^{2} \nabla_{F_{a}}^{\mathcal{L}} \theta^{a}, \\
\nabla_{E_{h}}^{g_{h}}, \omega^{j}=\nabla_{E_{i}}^{\mathcal{T}} \omega^{j}, & \nabla_{E_{i}}^{g_{h}}, \omega^{j}=\nabla_{E_{i}}^{\mathcal{L}} \omega^{j}, \\
\nabla_{F_{a}}^{g_{h}}, \omega^{i}=\nabla_{F_{a}}^{\mathcal{T}} \omega^{i}, & \nabla_{F_{a}}^{g_{h}} \omega^{i}=\nabla_{F_{a}}^{\mathcal{T}} \omega^{i}, \\
\nabla_{E_{i}}^{g_{h}, \mathcal{T}} \theta^{a}=h^{2} \nabla_{E_{i}}^{\mathcal{T}} \theta^{a}, & \nabla_{E_{i}}^{g_{h}, \mathcal{L}} \theta^{a}=h^{2} \nabla_{E_{i}}^{\mathcal{L}} \theta^{a} .
\end{array}
$$

Proof: We obtain the above relations using (4) and the fact that

$$
\begin{array}{ll}
\nabla_{U}^{\mathcal{T}} \theta^{b}\left(F_{a}\right)=-\theta^{b}\left(\nabla_{U}^{\mathcal{T}} F_{a}\right), & \nabla_{U}^{\mathcal{L}} \theta^{b}\left(E_{i}\right)=-\theta^{b}\left(\nabla_{U}^{\mathcal{T}} E_{i}\right), \\
\nabla_{U}^{\mathcal{T}} \omega^{i}\left(F_{a}\right)=-\omega^{i}\left(\nabla_{U}^{\mathcal{L}} F_{a}\right), & \nabla_{U}^{\mathcal{L}} \omega^{i}\left(E_{j}\right)=-\omega^{i}\left(\nabla_{U}^{\mathcal{L}} E_{j}\right),
\end{array}
$$

for any indices $i, j, a, b$ as above and arbitrary $U$, together with the corresponding relations for the metric $g_{h}$.

\section{Curvature tensor field and adiabatic limits}

Using the above relations, we are able to express now some of the the curvature operator components as polynomials in $h$. We denote by $R_{g_{h}, U, V}^{\mathcal{T}}$ and $R_{g_{h}, U, V}^{\mathcal{L}}$ the transversal and respectively the leafwise projection of the curvature operator $R_{g_{h}, U, V}=\nabla_{U}^{g_{h}} \nabla_{V}^{g_{h}}-\nabla_{V}^{g_{h}} \nabla_{U}^{g_{h}}-\nabla_{[U, V]}^{g_{h}}$ acting on differential forms.

First, using the notations adopted in the previous section, we obtain:

$$
\begin{aligned}
R_{g_{h}, f_{a}, f_{b}}^{\mathcal{T}} \alpha^{c}= & \nabla_{f_{a}}^{\mathcal{T}} \nabla_{F_{b}}^{\mathcal{T}} \theta^{c}-\nabla_{f_{b}}^{\mathcal{T}} \nabla_{F_{a}}^{\mathcal{T}} \theta^{c}-\nabla_{\left[F_{a}, F_{b}\right]^{\mathcal{T}}}^{\mathcal{T}} \theta^{c} \\
& +h^{2}\left(\nabla_{f_{a}}^{\mathcal{T}} \nabla_{F_{b}}^{\mathcal{L}} \theta^{c}-\nabla_{f_{b}}^{\mathcal{T}} \nabla_{F_{a}}^{\mathcal{L}} \theta^{c}-\nabla_{\left[F_{a}, F_{b}\right]^{\mathcal{L}}}^{\mathcal{T}} \theta^{c}\right) \\
= & R_{f_{a}, f_{b}}^{\perp} \theta^{c}+h^{2} R_{f_{a}, f_{b}}^{\mathcal{T}, 2} \theta^{c} .
\end{aligned}
$$

In the above calculations we denoted by $R^{\perp}$ the transversal curvature tensor field. Let us also consider the transversal curvature operator $\rho^{\perp}$, defined such that $g\left(\rho^{\perp}(U, V), W \wedge Z\right)=R^{\perp}(U, V, Z, W)$ for arbitrary local vector fields $U, V, W$ and $Z$ [MiRuTo].

In a similar manner,

$$
\begin{aligned}
R_{g_{h}, e_{i}, e_{j}}^{\mathcal{L}} \beta^{k}= & \nabla_{e_{i}}^{\mathcal{L}} \nabla_{E_{j}}^{\mathcal{L}} \omega^{k}-\nabla_{e_{j}}^{\mathcal{L}} \nabla_{E_{i}}^{\mathcal{L}} \omega^{k}-\nabla_{\left[E_{i}, E_{j}\right]}^{\mathcal{L}} \omega^{k} \\
& +h^{2}\left(\nabla_{e_{i}}^{\mathcal{L}} \nabla_{E_{j}}^{\mathcal{T}} \omega^{k}-\nabla_{e_{j}}^{\mathcal{L}} \nabla_{E_{i}}^{\mathcal{T}} \omega^{k}\right) \\
= & R_{e_{i}, e_{j}}^{\mathcal{F}} \beta^{k}+h^{2} R_{e_{i}, e_{j}}^{\mathcal{L}, 2} \beta^{k} .
\end{aligned}
$$


If we regard the leaves as immersed submanifolds, with the canonically induced metric, then the curvature operator $R^{\mathcal{F}}$ is in fact the curvature operator on leaves and it will be called in this paper the intrinsic curvature operator on leaves.

We calculate now the following two curvature terms:

$$
\begin{aligned}
R_{g_{h}, e_{i}, f_{a}}^{\mathcal{L}} \alpha^{c}= & h^{2}\left(\nabla_{e_{i}}^{\mathcal{L}} \nabla_{F_{a}}^{\mathcal{L}} \theta^{c}-\nabla_{f_{a}}^{\mathcal{L}} \nabla_{E_{i}}^{\mathcal{L}} \theta^{c}-\nabla_{\left[E_{i}, F_{a}\right]}^{\mathcal{L}} \theta^{c}\right. \\
& \left.+\nabla_{e_{i}}^{\mathcal{L}} \nabla_{F_{a}}^{\mathcal{T}} \theta^{c}\right)-h^{4}\left(\nabla_{f_{a}}^{\mathcal{L}} \nabla_{E_{i}}^{\mathcal{T}} \theta^{c}\right) \\
= & h^{2} R_{e_{i}, f_{a}}^{\mathcal{L}, 2} \alpha^{c}+h^{4} R_{e_{i}, f_{a}}^{\mathcal{L}, 4} \alpha^{c}, \\
R_{g_{h}, e_{i}, e_{j}}^{\mathcal{T}} \alpha^{c}= & h^{4}\left(\nabla_{e_{i}}^{\mathcal{T}} \nabla_{E_{j}}^{\mathcal{T}} \theta^{c}-\nabla_{e_{j}}^{\mathcal{T}} \nabla_{E_{i}}^{\mathcal{T}} \theta^{c}\right) \\
& +h^{2}\left(\nabla_{e_{i}}^{\mathcal{T}} \nabla_{E_{j}}^{\mathcal{L}} \theta^{c}-\nabla_{e_{j}}^{\mathcal{T}} \nabla_{E_{i}}^{\mathcal{L}} \theta^{c}-\nabla_{\left[E_{i}, E_{j}\right]}^{\mathcal{I}} \theta^{c}\right) \\
= & h^{4} R_{e_{i}, e_{j}}^{\mathcal{T}, 4} \alpha^{c}+h^{2} R_{e_{i}, e_{j}}^{\mathcal{T}, 2} \alpha^{c} .
\end{aligned}
$$

Due to the fact that $\left\{f_{a}, e_{i}\right\}$ is an orthonormal basis, we obtain

$$
\begin{aligned}
R_{g_{h}, e_{i}, f_{a}}^{\mathcal{L}} \alpha^{a}\left(e_{i}\right) & =h^{2} g_{h}\left(R_{g_{h}, e_{i}, f_{a}} f_{a}, e_{i}\right) \\
& =h^{2} R_{g_{h}}\left(e_{i}, f_{a}, f_{a}, e_{i}\right)
\end{aligned}
$$

and, as a consequence,

$$
R_{e_{i}, f_{a}}^{\mathcal{L}, 2} \alpha^{a}\left(e_{i}\right)=R^{0}\left(e_{i}, f_{a}, f_{a}, e_{i}\right)
$$

where by $R^{0}\left(e_{i}, f_{a}, f_{a}, e_{i}\right)$ we denote the $h^{0}$ coefficient of $g_{h}\left(R_{g_{h}, e_{i}, f_{a}} f_{a}, e_{i}\right)$.

Using the O'Neill calculations (see $[\mathrm{ON}]$ ), we can write:

$$
\begin{aligned}
g_{h}\left(R_{g_{h}, e_{i}, f_{a}} f_{a}, e_{i}\right)= & g_{h}\left(\left(\nabla_{f_{a}}^{g_{h}} T_{g_{h}}\right)_{e_{i}} e_{i}, f_{a}\right)-g_{h}\left(T_{g_{h}, e_{i}} f_{a}, T_{g_{h}, e_{i}} f_{a}\right) \\
& +g_{h}\left(\left(\nabla_{e_{i}}^{g_{h}} A_{g_{h}}\right)_{f_{a}} f_{a}, e_{i}\right)+g_{h}\left(A_{g_{h}, f_{a}} e_{i}, A_{g_{h}, f_{a}} e_{i}\right) .
\end{aligned}
$$

Considering the relations (4), with respect to the particular orthonormal basis $\left\{f_{a}, e_{i}\right\}$, we get

$$
\begin{aligned}
R^{0}\left(e_{i}, f_{a}, f_{a}, e_{i}\right)= & g\left(\left(\nabla_{f_{a}} T\right)_{e_{i}} e_{i}, f_{a}\right)+g\left(T_{e_{i}} f_{a}, T_{e_{i}} f_{a}\right) \\
& +g\left(\nabla_{e_{i}}\left(A_{f_{a}} f_{a}\right), e_{i}\right) .
\end{aligned}
$$


Finally, we have:

$$
\begin{aligned}
R_{g_{h}, f_{a}, f_{b}}^{\mathcal{L}} \alpha^{c}= & h^{2}\left(\nabla_{f_{a}}^{\mathcal{L}} \nabla_{F_{b}}^{\mathcal{L}} \theta^{c}-\nabla_{f_{b}}^{\mathcal{L}} \nabla_{F_{a}}^{\mathcal{L}} \theta^{c}-\nabla_{\left[F_{a}, F_{b}\right]}^{\mathcal{L}} \theta^{c}\right. \\
& \left.+\nabla_{f_{a}}^{\mathcal{L}} \nabla_{F_{b}}^{\mathcal{T}} \theta^{c}-\nabla_{f_{b}}^{\mathcal{L}} \nabla_{F_{a}}^{\mathcal{T}} \theta^{c}\right) \\
= & h^{2} R_{f_{a}, f_{b}}^{\mathcal{L}, 2} \alpha^{c}
\end{aligned}
$$

and

$$
\begin{aligned}
R_{g_{h}, e_{i}, f_{a}}^{\mathcal{T}} \alpha^{c}= & h^{2}\left(\nabla_{e_{i}}^{\mathcal{T}} \nabla_{F_{a}}^{\mathcal{T}} \theta^{c}-\nabla_{f_{a}}^{\mathcal{T}} \nabla_{E_{i}}^{\mathcal{T}} \theta^{c}-\nabla_{\left[E_{i}, F_{a}\right]}^{\mathcal{T}} \theta^{c}\right. \\
& \left.+\nabla_{e_{i}}^{\mathcal{T}} \nabla_{F_{a}}^{\mathcal{L}} \theta^{c}-\nabla_{f_{a}}^{\mathcal{T}} \nabla_{E_{i}}^{\mathcal{L}} \theta^{c}\right) \\
= & h^{2} R_{e_{i}, f_{a}}^{\mathcal{T}, 2} \alpha^{c} .
\end{aligned}
$$

Remark $1 R_{g_{h}, f_{a}, f_{b}}^{\mathcal{L}} \alpha^{c}$ and $R_{g_{h}, e_{i}, f_{a}}^{\mathcal{T}} \alpha^{c}$ have not an $h^{0}$ coefficient.

\section{Weitzenböck formula and adiabatic limits}

Let us now consider the classical Weitzenböck formula (see e.g. [Pet]). We take an orthonormal frame field $\left\{\mathcal{E}_{i}\right\}$ in the neighborhood of an arbitrary point $x \in M$ which induces an orthonormal basis $\left\{\epsilon_{i}\right\}$ at $x$ such that $\nabla_{\epsilon_{i}} \mathcal{E}_{j}=$ 0 , with $1 \leq i, j \leq n$. If $\left\{\Theta^{i}\right\}$ and $\left\{\theta^{i}\right\}$ are the dual coframes for $\left\{\mathcal{E}_{i}\right\}$ and $\left\{\epsilon_{i}\right\}$ respectively, considering that $d=\sum_{i} \Theta^{i} \wedge \nabla_{\mathcal{E}_{i}}$ and $\delta=-\sum_{i} i_{\mathcal{E}_{i}} \nabla_{\mathcal{E}_{i}}$, we can express the Laplace operator:

$$
\begin{aligned}
\Delta=d \delta+\delta d & =-\sum_{i, j} \theta^{i} \wedge \nabla_{\epsilon_{i}}\left(i_{\mathcal{E}_{j}} \nabla_{\mathcal{E}_{j}}\right)-\sum_{j, i} i_{\epsilon_{j}} \nabla_{\epsilon_{j}}\left(\Theta^{i} \wedge \nabla_{\mathcal{E}_{i}}\right) \\
& =-\sum_{i} \nabla_{\epsilon_{i}} \nabla_{\epsilon_{i}}+\sum_{i, j} \theta^{i} \wedge i_{\epsilon_{j}}\left(\nabla_{\epsilon_{j}} \nabla_{\mathcal{E}_{i}}-\nabla_{\epsilon_{i}} \nabla_{\mathcal{E}_{j}}\right) \\
& =\nabla^{*} \nabla+\sum_{i, j} \theta^{i} \wedge i_{\epsilon_{j}} R_{\epsilon_{j}, \epsilon_{i}} \\
& =\nabla^{*} \nabla+\sum_{i<j} \epsilon_{i} \cdot \epsilon_{j} \cdot R_{\epsilon_{i}, \epsilon_{j}},
\end{aligned}
$$

where dot stands for Clifford multiplication. In the following we denote the 0-th order operator $\sum_{i<j} \epsilon_{i} \cdot \epsilon_{j} \cdot R_{\epsilon_{i}, \epsilon_{j}}=\sum_{i, j} \theta^{i} \wedge i_{\epsilon_{j}} R_{\epsilon_{j}, \epsilon_{i}}$ by $K$. 
If we choose a foliated chart $\mathcal{U}$ on $M$, then

$$
\Omega^{u, v}(\mathcal{U})=\Omega^{u}\left(\mathcal{U} / \mathcal{F}_{\mathcal{U}}\right) \wedge \Omega^{0, v}(\mathcal{U}) \equiv \Omega^{u}\left(\mathcal{U} / \mathcal{F}_{\mathcal{U}}\right) \otimes \Omega^{0, v}(\mathcal{U}),
$$

according to [AlKo2]. Then, if we take $\alpha \in \Omega^{u}\left(\mathcal{U} / \mathcal{F}_{\mathcal{U}}\right)$ and $\beta \in \Omega^{0, v}(\mathcal{U})$, we can evaluate the above operator acting on differential forms of the type $\alpha \wedge \beta$, the general formula being easy to obtain by linearity.

We consider now the covariant derivative $\nabla$ induced on $\Omega^{u, v}$, with $u$ and $v$ satisfying $u+v=r$ :

$$
\nabla: \Omega^{u, v} \longrightarrow C^{\infty}\left(T M^{*}\right) \otimes C^{\infty}\left(\Lambda^{r} T M^{*}\right) .
$$

Now we define the following six differential operators:

$$
\begin{array}{ll}
\nabla_{\mathcal{T}, 0,0}=\left(\mathrm{pr}^{\mathcal{T}} \otimes \pi_{u, v}\right) \circ \nabla, & \nabla_{\mathcal{L}, 0,0}=\left(\mathrm{pr}^{\mathcal{L}} \otimes \pi_{u, v}\right) \circ \nabla, \\
\nabla_{\mathcal{T},-1,1}=\left(\mathrm{pr}^{\mathcal{T}} \otimes \pi_{u-1, v+1}\right) \circ \nabla, & \nabla_{\mathcal{L},-1,1}=\left(\mathrm{pr}^{\mathcal{L}} \otimes \pi_{u-1, v+1}\right) \circ \nabla, \\
\nabla_{\mathcal{T}, 1,-1}=\left(\mathrm{pr}^{\mathcal{T}} \otimes \pi_{u+1, v-1}\right) \circ \nabla, & \nabla_{\mathcal{L}, 1,-1}=\left(\mathrm{pr}^{\mathcal{L}} \otimes \pi_{u+1, v-1}\right) \circ \nabla .
\end{array}
$$

where $\pi_{u, v}, \pi_{u-1, v+1}$ and $\pi_{u+1, v-1}$ are canonical projections induced by the bigrading.

The above operators can be naturally extended from $\Omega^{u, v}$ to $\Omega$.

Remark 2 It follows easily that:

$$
\begin{array}{r}
\nabla \omega=\nabla_{\mathcal{T}, 0,0} \omega+\nabla_{\mathcal{L}, 0,0} \omega+\nabla_{\mathcal{T},-1,1} \omega \\
+\nabla_{\mathcal{L},-1,1} \omega+\nabla_{\mathcal{T}, 1,-1} \omega+\nabla_{\mathcal{L}, 1,-1} \omega .
\end{array}
$$

Considering also the bigrading, we get:

$$
K=K_{-2,2}+K_{-1,1}+K_{0,0}+K_{1,-1}+K_{2,-2} .
$$

Note that

$$
\begin{aligned}
\sum_{i, j} \theta^{i} \wedge i_{\epsilon_{j}} R_{\epsilon_{j}, \epsilon_{i}}(\alpha \wedge \beta)= & \sum_{i, j} \theta^{i} \wedge i_{\epsilon_{j}} R_{\epsilon_{j}, \epsilon_{i}} \alpha \wedge \beta+\alpha \wedge \sum_{i, j} \theta^{i} \wedge i_{\epsilon_{j}} R_{\epsilon_{j}, \epsilon_{i}} \beta \\
& +\sum_{i, j} \theta^{i} \wedge i_{\epsilon_{j}} \alpha \wedge R_{\epsilon_{j}, \epsilon_{i}} \beta+\sum_{i, j} R_{\epsilon_{j}, \epsilon_{i}} \alpha \wedge \theta^{i} \wedge i_{\epsilon_{j}} \beta .
\end{aligned}
$$

Using the orthonormal frames $\left\{f_{a}, e_{i}\right\}$ and $\left\{\alpha^{a}, \beta^{i}\right\}$ at the arbitrary point $x$, we obtain:

$$
K_{-2,2}(\alpha \wedge \beta)=\sum_{i, a} \beta^{i} \wedge R_{f_{a}, e_{i}}^{\mathcal{L}} i_{f_{a}} \alpha \wedge \beta
$$




$$
\begin{aligned}
& K_{-1,1}(\alpha \wedge \beta)=\sum_{a, b} \alpha^{a} \wedge R_{f_{b}, f_{a}}^{\mathcal{L}} i_{f_{b}} \alpha \wedge \beta+\sum_{i, a} \beta^{i} \wedge i_{f_{a}} \alpha \wedge R_{f_{a}, e_{i}}^{\mathcal{L}} \beta \\
& +\sum_{i, a} \beta^{i} \wedge i_{f_{a}} R_{f_{a}, e_{i}}^{\mathcal{T}} \alpha \wedge \beta+\sum_{i, j} R_{e_{i}, e_{j}}^{\mathcal{L}} \alpha \wedge \beta^{j} \wedge i_{e_{i}} \beta \\
& +\sum_{i, j} \beta^{i} \wedge i_{e_{j}} R_{e_{j}, e_{i}}^{\mathcal{L}} \alpha \wedge \beta \\
& K_{0,0}(\alpha \wedge \beta)=\sum_{a, b} \alpha^{a} \cdot \alpha^{b} \cdot R_{f_{a}, f_{b}}^{\mathcal{T}} \alpha \otimes \beta+\sum_{a, b} \alpha^{a} \wedge i_{f_{b}} \alpha \otimes R_{f_{b}, f_{a}}^{\mathcal{L}} \beta \\
& +\sum_{i, a} \beta^{i} \wedge i_{f_{a}} \alpha \otimes R_{f_{a}, e_{i}}^{\mathcal{T}} \beta+\alpha \otimes \sum_{i, a} \beta^{i} \wedge i_{f_{a}} R_{f_{a}, e_{i}}^{\mathcal{T}} \beta \\
& +\sum_{i, a} \alpha^{a} \wedge i_{e_{i}} R_{e_{i}, f_{a}}^{\mathcal{L}} \alpha \otimes \beta+\sum_{i, a} R_{e_{i}, f_{a}}^{\mathcal{L}} \alpha \otimes \alpha^{a} \wedge i_{e_{i}} \beta \\
& +\sum_{i, j} R_{e_{i}, e_{j}}^{\mathcal{T}} \alpha \otimes \beta^{i} \wedge i_{e_{j}} \beta+\alpha \otimes \sum_{i, j} \beta^{i} \cdot \beta^{j} \cdot R_{e_{i}, e_{j}}^{\mathcal{L}} \beta \\
& K_{1-1}(\alpha \wedge \beta)=\sum_{a, b} \alpha^{a} \wedge i_{f_{b}} \alpha \wedge R_{f_{b}, f_{a}}^{\mathcal{T}} \beta+\alpha \wedge \sum_{a, b} \alpha^{a} \wedge i_{f_{b}} R_{f_{b}, f_{a}}^{\mathcal{T}} \beta \\
& +\sum_{i, a} R_{e_{i}, f_{a}}^{\mathcal{T}} \alpha \wedge \alpha^{a} \wedge i_{e_{i}} \beta+\alpha \wedge \sum_{i, a} \alpha^{a} \wedge i_{e_{i}} R_{e_{i}, f_{a}}^{\mathcal{L}} \beta \\
& +\alpha \wedge \sum_{i, j} \beta^{j} \wedge R_{e_{i}, e_{j}}^{\mathcal{T}} i_{e_{i}} \beta, \\
& K_{-2,2}(\alpha \wedge \beta)=\alpha \wedge \sum_{i, a} \alpha^{a} \wedge R_{e_{i}, f_{a}}^{\mathcal{T}} i_{e_{i}} \beta .
\end{aligned}
$$

Using the rescaling homomorphism mentioned in the introductory section, let us now define

$$
\begin{aligned}
\Delta_{h} & :=\Theta_{h} \Delta_{g_{h}} \Theta_{h}^{-1}, \\
\nabla^{h} & :=\Theta_{h} \nabla^{g_{h}} \Theta_{h}^{-1}
\end{aligned}
$$

and

$$
K^{h}:=\Theta_{h} K_{g_{h}} \Theta_{h}^{-1} .
$$

Now, applying (13) for $\Theta_{h}^{-1} \omega$, where $\omega \in \Omega^{r}$ and using that $\Theta_{h}$ is in fact an isometry of Riemannian vector bundles, we obtain the formula

$$
\left\langle\Delta_{h} \omega, \omega\right\rangle=\left\langle\nabla^{h} \omega, \nabla^{h} \omega\right\rangle+\left\langle K^{h} \omega, \omega\right\rangle .
$$


We will study each term of (22), our goal being to express all terms as polynomials in $h$. For the first one we refer to [AlKo2]:

$$
\begin{aligned}
\left\langle\Delta_{h} \omega, \omega\right\rangle= & \left\langle\Delta_{0} \omega, \omega\right\rangle+h\left\langle\left(D_{\perp} D_{0}+D_{0} D_{\perp}\right) \omega, \omega\right\rangle \\
& +h^{2}\left(\left\langle\left(D_{0} F+F D_{0}\right) \omega, \omega\right\rangle+\left\langle\Delta_{\perp} \omega, \omega\right\rangle\right) \\
& +h^{3}\left\langle\left(D_{\perp} F+F D_{\perp}\right) \omega, \omega\right\rangle+h^{4}\left\langle F^{2} \omega, \omega\right\rangle,
\end{aligned}
$$

where $F$ is the 0 -th order operator $d_{2,-1}+\delta_{-2,1}, D_{0}:=d_{0,1}+\delta_{0,-1}, \Delta_{0}=$ $D_{0} \circ D_{0}$ and $D_{\perp}:=d_{1,0}+\delta_{-1,0}$ (see $[\mathrm{AlKo} 2]$ ).

Let us study now the second term:

$$
\begin{aligned}
\nabla^{h} & =\Theta_{h} \nabla^{g_{h}} \Theta_{h}^{-1} \\
& =\nabla_{\mathcal{T}, 0,0}^{h}+\nabla_{\mathcal{L}, 0,0}^{h}+\nabla_{\mathcal{T},-1,1}^{h}+\nabla_{\mathcal{L},-1,1}^{h}+\nabla_{\mathcal{T}, 1,-1}^{h}+\nabla_{\mathcal{L}, 1,-1}^{h} .
\end{aligned}
$$

Considering the change of the bigrading, we get the following relations:

$$
\begin{array}{ll}
\nabla_{\mathcal{T}, 0,0}^{h}=h \nabla_{\mathcal{T}, 0,0}^{g_{h}}, & \nabla_{\mathcal{L}, 0,0}^{h}=\nabla_{\mathcal{L}, 0,0}^{g_{h}}, \\
\nabla_{\mathcal{T},-1,1}^{h}=\nabla_{\mathcal{T},-1,1}^{g_{h}}, & \nabla_{\mathcal{L},-1,1}^{h}=h^{-1} \nabla_{\mathcal{L},-1,1}^{g_{h}}, \\
\nabla_{\mathcal{T}, 1,-1}^{h}=h^{2} \nabla_{\mathcal{T}, 1,-1}^{g_{h}}, & \nabla_{\mathcal{L}, 1,-1}^{h}=h \nabla_{\mathcal{L}, 1,-1}^{g_{h}} .
\end{array}
$$

Remark 3 In accordance with (5), the operators $\nabla_{\mathcal{T}, 0,0}^{g_{h}}, \nabla_{\mathcal{L}, 0,0}^{g_{h}}, \nabla_{\mathcal{T},-1,1}^{g_{h}}$, $\nabla_{\mathcal{L},-1,1}^{g_{h}}, \quad \nabla_{\mathcal{T}, 1,-1}^{g_{h}}$ and $\nabla_{\mathcal{L}, 1,-1}^{g_{h}}$ - induced by the metric $g_{h}$, are homogeneous with respect to $h$ when they act on $\Omega^{u}\left(\mathcal{U} / \mathcal{F}_{\mathcal{U}}\right)$ and $\Omega^{0, v}(\mathcal{U})$.

Using (5), (14), (23) and the above remark we write all these operators only using the Levi-Civita connection associated to $g$ and the adiabatic parameter $h$.

For the first two operators we have:

$$
\begin{aligned}
& \nabla_{\mathcal{T}, 0,0}^{h}(\alpha \wedge \beta)=h\left(\nabla_{\mathcal{T}, 0,0}^{g_{h}} \alpha \otimes \beta+\alpha \otimes \nabla_{\mathcal{T}, 0,0}^{g_{h}} \beta\right)=h \nabla_{\mathcal{T}, 0,0}(\alpha \wedge \beta) . \\
& \nabla_{\mathcal{L}, 0,0}^{h}(\alpha \wedge \beta)=\nabla_{\mathcal{L}, 0,0}^{g_{h}}(\alpha \wedge \beta)=\alpha \otimes \nabla_{\mathcal{L}, 0,0} \beta+h^{2} \nabla_{\mathcal{L}, 0,0} \alpha \otimes \beta .
\end{aligned}
$$

In the following we denote $\operatorname{id}_{\Omega^{u}\left(\mathcal{U} / \mathcal{F}_{\mathcal{U}}\right)} \otimes \nabla_{\mathcal{L}, 0,0}$ by $\nabla_{\mathcal{L}, 0,0}^{0}$ and $\nabla_{\mathcal{L}, 0,0} \otimes \mathrm{id}_{\Omega^{0, \cdot}(\mathcal{U})}$ by $\nabla_{\mathcal{L}, 0,0}^{2}$.

For the last four operators we obtain:

$$
\begin{aligned}
& \nabla_{\mathcal{T},-1,1}^{h}(\alpha \wedge \beta)=\nabla_{\mathcal{T},-1,1}^{g_{h}}(\alpha \wedge \beta)=h^{2} \nabla_{\mathcal{T},-1,1} \alpha \wedge \beta=h^{2} \nabla_{\mathcal{T},-1,1}(\alpha \wedge \beta), \\
& \nabla_{\mathcal{L},-1,1}^{h}(\alpha \wedge \beta)=h^{-1} \nabla_{\mathcal{L},-1,1}^{g_{h}}(\alpha \wedge \beta)=h \nabla_{\mathcal{L},-1,1} \alpha \wedge \beta=h \nabla_{\mathcal{L},-1,1}(\alpha \wedge \beta), \\
& \nabla_{\mathcal{T}, 1,-1}^{h}(\alpha \wedge \beta)=h^{2} \nabla_{\mathcal{T}, 1,-1}^{h}(\alpha \wedge \beta)=h^{2} \alpha \wedge \nabla_{\mathcal{T}, 1,-1} \beta=h^{2} \nabla_{\mathcal{T}, 1,-1}(\alpha \wedge \beta), \\
& \nabla_{\mathcal{L}, 1,-1}^{h}(\alpha \wedge \beta)=h \nabla_{\mathcal{L}, 1,-1}^{h}(\alpha \wedge \beta)=h \alpha \wedge \nabla_{\mathcal{L}, 1,-1} \beta=h \nabla_{\mathcal{L}, 1,-1}(\alpha \wedge \beta) .
\end{aligned}
$$

Summing up, we get: 
Proposition 4 a) The rescaled covariant derivative has the following components:

$$
\begin{array}{ll}
\nabla_{\mathcal{T}, 0,0}^{h}=h \nabla_{\mathcal{T}, 0,0}, & \nabla_{\mathcal{L}, 0,0}^{h}=\nabla_{\mathcal{L}, 0,0}^{0}+h^{2} \nabla_{\mathcal{L}, 0,0}^{2}, \\
\nabla_{\mathcal{T},-1,1}^{h}=h^{2} \nabla_{\mathcal{T},-1,1}, & \nabla_{\mathcal{L},-1,1}^{h}=h \nabla_{\mathcal{L},-1,1}, \\
\nabla_{\mathcal{T}, 1,-1}^{h}=h^{2} \nabla_{\mathcal{T}, 1,-1}, & \nabla_{\mathcal{L}, 1,-1}^{h}=h \nabla_{\mathcal{L}, 1,-1} .
\end{array}
$$

b) The following polynomial description is valid:

$$
\begin{aligned}
\nabla^{h} \omega= & \nabla_{\mathcal{L}, 0,0}^{0} \omega+h\left(\nabla_{\mathcal{T}, 0,0} \omega+\nabla_{\mathcal{L},-1,1}+\nabla_{\mathcal{L}, 1,-1} \omega \omega\right) \\
& +h^{2}\left(\nabla_{\mathcal{L}, 0,0}^{2} \omega+\nabla_{\mathcal{T},-1,1} \omega+\nabla_{\mathcal{T}, 1,-1} \omega\right) .
\end{aligned}
$$

Finally, we express $\left\|\nabla^{h} \omega\right\|^{2}$ as a polynomial in $h$ :

$$
\begin{aligned}
\left\|\nabla^{h} \omega\right\|^{2}= & \left\|\nabla_{\mathcal{L}, 0,0}^{0} \omega\right\|^{2}+2 h\left(\left\langle\nabla_{\mathcal{L}, 0,0}^{0} \omega, \nabla_{\mathcal{L}, 1,-1} \omega\right\rangle+\left\langle\nabla_{\mathcal{L}, 0,0}^{0} \omega, \nabla_{\mathcal{L},-1,1} \omega\right\rangle\right) \\
& +h^{2}\left(2\left\langle\nabla_{\mathcal{L}, 0,0}^{0} \omega, \nabla_{\mathcal{L}, 0,0}^{2} \omega\right\rangle+2\left\langle\nabla_{\mathcal{L}, 1,-1} \omega, \nabla_{\mathcal{L},-1,1} \omega\right\rangle+\left\|\nabla_{\mathcal{T}, 0,0} \omega\right\|^{2}\right. \\
& \left.+\left\|\nabla_{\mathcal{L}, 1,-1} \omega\right\|^{2}+\left\|\nabla_{\mathcal{L},-1,1} \omega\right\|^{2}\right)+o\left(h^{2}\right) .
\end{aligned}
$$

Now we investigate the last term of (22). The above formulas concerning the curvature expression (see (6)-(12)) allow us to express $K^{h}$ as a polynomial in $h$, that is

$$
K^{h}=\sum_{i=0}^{4} h^{i} \cdot K^{i}
$$

and in accordance with the bigrading, that means:

$$
K^{i}=K_{-2,2}^{i}+K_{-1,1}^{i}+K_{0,0}^{i}+K_{1,-1}^{i}+K_{2,-2}^{i}
$$

for $0 \leq i \leq 4$

Some of the above operators will be of particular interest for us in what follows.

Remark 4 Let us consider (22) for $\omega^{u, v} \in \Omega^{u, v}$. Both sides of the equality can be written as polynomials in $h$; as leafwise Laplacian $\Delta_{0}$ does not change the bigrading of a differential form, if we write only the coefficients of $h^{0}$, we obtain:

$$
\left\langle\Delta_{0} \omega^{u, v}, \omega^{u, v}\right\rangle=\left\|\nabla_{\mathcal{L}, 0,0}^{0} \omega^{u, v}\right\|^{2}+\left\langle K_{0,0}^{0} \omega^{u, v}, \omega^{u, v}\right\rangle .
$$

The above relation is a Weitzenböck type formula for the leafwise Laplacian $\Delta_{0}$. In fact it is an extension of the result stated in [AlTo], p. 456; for $\omega^{0, .} \in \Omega^{0, .}$, the above formula becomes the Weitzenböck type formula provided by the authors in that paper. 
In order to obtain vanishing results we study the $K_{0,0}^{0}$ operator. This is exactly the $h^{0}$ term of the operator $K_{0,0}^{h}$.

Considering $\left\{f_{a}^{h}, e_{i}^{h}\right\}$ and $\left\{f_{a}, e_{i}\right\}$ two orthonormal bases for the $g_{h}$ and $g$ metric respectively, and the dual bases $\left\{\alpha^{h, a}, \beta^{h, i}\right\}$ and $\left\{\alpha^{a}, \beta^{i}\right\}$ with $1 \leq$ $a \leq q, 1 \leq i \leq p$, these bases are subject to the relations:

$$
\begin{cases}f_{a}^{h}=h f_{a}, & e_{i}^{h}=e_{i} \\ \alpha^{h, a}=h^{-1} \alpha^{a}, & \beta^{h, i}=\beta^{i}\end{cases}
$$

Using (19), we obtain:

$$
\begin{aligned}
K_{0,0}^{h}(\alpha \wedge \beta)= & h^{2}\left\{\sum_{a, b} \alpha^{a} \wedge i_{f_{b}} R_{g_{h}, f_{b}, f_{a}}^{\mathcal{T}} \alpha \otimes \beta+\sum_{a, b} \alpha^{a} \wedge i_{f_{b}} \alpha \otimes R_{g_{h}, f_{b}, f_{a}}^{\mathcal{L}} \beta\right. \\
& \left.+\sum_{i, a} \beta^{i} \wedge i_{f_{a}} \alpha \otimes R_{g_{h}, f_{a}, e_{i}}^{\mathcal{T}} \beta+\alpha \otimes \sum_{a, i} \beta^{i} \wedge i_{f_{a}} R_{g_{h}, f_{a}, e_{i}}^{\mathcal{T}} \beta\right\} \\
& +\sum_{a, i} \alpha^{a} \wedge i_{e_{i}} R_{g_{h}, e_{i}, f_{a}}^{\mathcal{L}} \alpha \otimes \beta+\sum_{a, i} R_{g_{h}, e_{i}, f_{a}}^{\mathcal{L}} \alpha \otimes \alpha^{a} \wedge i_{e_{i}} \beta \\
& +\sum_{i, j} R_{g_{h}, e_{i}, e_{j}}^{\mathcal{T}} \alpha \otimes \beta^{i} \wedge i_{e_{j}} \beta+\alpha \otimes \sum_{i, j} \beta^{i} \wedge i_{e_{j}} R_{g_{h}, e_{j}, e_{i}}^{\mathcal{L}} \beta .
\end{aligned}
$$

In each one of the above eight terms, using the relations (6)-(12) we can calculate the corresponding $h^{0}$ coefficient. We observe that only the last term contains such component, so we finally get:

$$
\begin{aligned}
K_{0,0}^{0}(\alpha \wedge \beta) & =\alpha \otimes \sum_{i, j} \beta^{i} \wedge i_{e_{j}} R_{e_{j}, e_{i}}^{\mathcal{F}} \beta \\
& =\alpha \otimes \sum_{i<j} \beta^{i} \cdot \beta^{j} \cdot R_{e_{i}, e_{j}}^{\mathcal{F}} \beta .
\end{aligned}
$$

\section{Vanishing conditions for the spectral se- quence of a Riemannian foliation}

According to Proposition 1, assuming that $\omega \in \mathcal{H}_{2}$, it follows the existence of a family of smooth forms $\left(\omega_{h}\right)_{h>0}, \omega_{h} \rightarrow \omega$ in $L^{2}$-norm as $h \downarrow 0$, and $\left\langle\Delta_{h} \omega_{h}, \omega_{h}\right\rangle \in o\left(h^{2}\right)$. Under these circumstances, we can reshape the 
"rescaled" Weitzenböck formula (22)

$$
\begin{aligned}
\left\langle\Delta_{h} \omega_{h}, \omega_{h}\right\rangle= & \left\|\nabla^{h} \omega_{h}\right\|^{2}+\left\langle K^{0} \omega_{h}, \omega_{h}\right\rangle+h\left\langle K^{1} \omega_{h}, \omega_{h}\right\rangle \\
& +h^{2}\left\langle K^{2} \omega_{h}, \omega_{h}\right\rangle+o\left(h^{2}\right),
\end{aligned}
$$

so we can prove now Theorem 1 .

Proof of Theorem 1. Let $\omega^{u, v} \in \mathcal{H}_{2}^{u, v}, \omega^{u, v} \neq 0$. As above, we consider a family of differential forms $\omega_{h}, h>0$, such that $\omega_{h} \rightarrow \omega^{u, v}$ in the $L^{2}$ norm as $h \downarrow 0$, and $\left\langle\Delta_{h} \omega_{h}, \omega_{h}\right\rangle \in o\left(h^{2}\right)$. We assumed that $\left\langle K^{0} \omega_{h}, \omega_{h}\right\rangle \geq 0$, $\left\langle K^{1} \omega_{h}, \omega_{h}\right\rangle \geq 0$, and also that there is a constant $\mathbf{c}>0$ such that $\left\langle K^{2} \omega_{h}, \omega_{h}\right\rangle>$ c $\left\|\omega_{h}\right\|^{2}$, as $M$ is a compact manifold. Then we get

$$
\begin{aligned}
\left\langle\Delta_{h} \omega_{h}, \omega_{h}\right\rangle= & \left\|\nabla^{h} \omega_{h}\right\|^{2}+h^{0}\left\langle K^{0} \omega_{h}, \omega_{h}\right\rangle+h^{1}\left\langle K^{1} \omega_{h}, \omega_{h}\right\rangle \\
& +h^{2}\left\langle K^{2} \omega_{h}, \omega_{h}\right\rangle+o\left(h^{2}\right) \\
> & \mathbf{c}\left\|\omega_{h}\right\|^{2}+o\left(h^{2}\right),
\end{aligned}
$$

since $\left\|\nabla_{h} \omega_{h}\right\|^{2}+h^{0}\left\langle K^{0} \omega_{h}, \omega_{h}\right\rangle+h^{1}\left\langle K^{1} \omega_{h}, \omega_{h}\right\rangle \geq 0$, and, as a consequence, $\left\langle\Delta_{h} \omega_{h}, \omega_{h}\right\rangle \notin o\left(h^{2}\right)$. The contradiction comes from the fact that we assumed $\omega^{u, v} \neq 0$. Then $\mathcal{H}_{2}^{u, v}=0$, and also $E_{2}^{u, v}=0$, considering the isomorphism that exists between these topological vector spaces.

Proof of Theorem 2. We study the particular case when $\omega=\omega^{q, 0} \in \mathcal{H}^{q, 0}$.

First of all, if $\omega^{q, 0} \in \mathcal{H}^{q, 0}$, let $\left(\omega_{h}^{q, 0}\right)_{h>0}, \omega_{h}^{q, 0}=\omega^{q, 0}+h \beta_{h}^{q-1,1}, \beta_{h}^{q-1,1} \in$ $\Omega^{q-1,1}$ be the sequence obtained as above (see also [AlKo2]); under these circumstances we can refine the Weitzenböck type formula (26):

$$
\begin{aligned}
& \left\langle\Delta_{h}\left(\omega^{q, 0}+h \beta_{h}^{q-1,1}\right), \omega^{q, 0}+h \beta_{h}^{q-1,1}\right\rangle \\
= & \left\|\nabla^{h}\left(\omega^{q, 0}+h \beta_{h}^{q-1,1}\right)\right\|^{2}+\left\langle K_{0,0}^{0} \omega^{q, 0}, \omega^{q, 0}\right\rangle \\
& +h^{2}\left\{2\left\langle K_{-1,1}^{1} \omega^{q, 0}, \beta_{h}^{q-1,1}\right\rangle+\left\langle K_{0,0}^{0} \beta_{h}^{q-1,1}, \beta_{h}^{q-1,1}\right\rangle\right. \\
& \left.+\left\langle K_{0,0}^{2} \omega^{q, 0}, \omega^{q, 0}\right\rangle\right\}+o\left(h^{2}\right) .
\end{aligned}
$$

In the relation (16), identifying the corresponding coefficient of $h^{0}$ and $h^{2}$ when writing $K^{h}$ as a polynomial in $h$, and considering also the relations (18)-(19) we finally end up with the necessary formulas for the operators $K_{0,0}^{0}$ and $K_{-1,1}^{1}$. First of all we observe that $K_{0,0}^{0} \alpha^{1} \wedge . . \wedge \alpha^{q}=0$; as $K_{0,0}^{0}$ is a linear operator, we extend the result for any $\omega^{u, v} \in \Omega^{u, v}$. 
Let us now study this operator when acting on $\beta^{q-1,1}=\alpha^{i_{1}} \wedge . . \wedge \alpha^{i_{q-1}} \otimes$ $\beta^{k} \in \Omega^{q-1,1}$.

$$
\begin{aligned}
K_{0,0}^{0} \beta^{q-1,1} & =\alpha^{i_{1}} \wedge . . \wedge \alpha^{i_{q-1}} \otimes \sum_{i<j} \beta^{i} \cdot \beta^{j} \cdot R_{e_{i}, e_{j}}^{\mathcal{F}} \beta^{k} \\
& =\alpha^{i_{1}} \wedge . . \wedge \alpha^{i_{q-1}} \otimes \operatorname{Ric}^{\mathcal{F}} \beta^{k} \\
& =\operatorname{Ric}^{\mathcal{F}} \beta^{q-1,1}
\end{aligned}
$$

From here it follows that $\left\langle K_{0,0}^{0} \beta_{h}^{q-1,1}, \beta_{h}^{q-1,1}\right\rangle=\left\langle R i c^{\mathcal{F}} \beta_{h}^{q-1,1}, \beta_{h}^{q-1,1}\right\rangle \geq 0$ if and only if the tangent Ricci operator is non-negative.

Now, if we evaluate the action of the operator $K_{0,0}^{2}$ on $\omega^{q, 0}=\alpha^{1} \wedge . . \wedge \alpha^{q} \in$ $\Omega^{q, 0}$, the following relation is obtained:

$$
\begin{aligned}
K_{0,0}^{2} \omega^{q, 0} & =\sum_{a, b} \alpha^{a} \wedge i_{f_{b}} R_{f_{b}, f_{a}}^{\mathcal{T}, 2} \omega^{q, 0}+\sum_{i, a} \alpha^{a} \wedge i_{e_{i}} R_{e_{i}, f_{a}}^{\mathcal{L}, 2} \omega^{q, 0} \\
& =\sum_{i, a} \alpha^{a} \wedge i_{e_{i}} R_{e_{i}, f_{a}}^{\mathcal{L}, 2} \omega^{q, 0} \\
& =\sum_{i, a}(-1)^{a-1} \alpha^{a} \wedge \alpha^{1} \wedge . . \wedge \alpha^{a-1} \wedge R_{e_{i}, f_{a}}^{\mathcal{L}, 2} \alpha^{a}\left(e_{i}\right) \wedge . \wedge \alpha^{q} \\
& =\sum_{i, a} R^{0}\left(e_{i}, f_{a}, f_{a}, e_{i}\right) \alpha^{1} \wedge . . \wedge \alpha^{q} \\
& =\text { scal }_{\text {mixed }}^{0} \alpha^{1} \wedge . . \wedge \alpha^{q},
\end{aligned}
$$

where $s c a l_{\text {mixed }}^{0}$ is the mixed scalar curvature corresponding to the $R^{0}$-mixed curvature tensor field (see (10)). In the above calculations we have used the fact that, in general, the operator $K$ vanishes when it acts on a differential form of top dimensional degree.

Finally, we present the operator $K_{-1,1}^{1}$ acting on differential forms of the type $\omega^{q, 0}$, with $\omega^{q, 0} \in \Omega^{q, 0}$.

$$
\begin{aligned}
K_{-1,1}^{1} \omega^{q, 0}= & \sum_{a, b} \alpha^{a} \wedge R_{f_{b}, f_{a}}^{\mathcal{L}, 0} i_{f_{b}} \omega^{q, 0}+\sum_{i, a} \beta^{i} \wedge i_{f_{a}} R_{f_{a}, e_{i}}^{\mathcal{T}, 0} \omega^{q, 0} \\
& +\sum_{i, j} \beta^{i} \wedge i_{e_{j}} R_{e_{j}, e_{i}}^{\mathcal{L}, 2} \omega^{q, 0} \\
= & \sum_{i, j} \beta^{i} \wedge i_{e_{j}} R_{e_{j}, e_{i}}^{\mathcal{L}, 2} \omega^{q, 0}
\end{aligned}
$$


in accordance with Remark 1.

Now, as $\mathcal{H}_{b}^{q}$ and $E_{2}^{q, 0}$ are isomorphic topological vector spaces, the vanishing result stated in Theorem 2 is just a straightforward application of the above results .

Remark 5 There are very interesting connections between the term $H_{b}^{q}(\mathcal{F})$ of the basic cohomology and the property of being taut (see e.g. [Al]). If the dimension of the leaves is equal to 1, then the foliation is geodesible if and only if $H_{b}^{q}(\mathcal{F}) \neq 0$ (see $[M o S e]$ ). In this case the leaves are curves and the leafwise Riemannian tensor field $R^{\mathcal{L}}$ vanishes, as well as the other "mixed" components of the curvature tensor field that imply more than one tangent to the leaves vector field, so we finally obtain Corollary 1.

Remark 6 The Riemannian flows defined on a 3-dimensional manifold were already classified by $Y$. Carrière in [Car]; the author presents an example of non-geodesible Riemannian flow using a direct computation. Following the same idea, D. Dominguez gives another example (see [Do]). The above results might help us to find non-geodesible Riemannian flows and, in general, to study Riemannian foliations in higher dimension.

In the final part of this paper we investigate the special case of a Riemannian foliation which locally can be described as a product of two Riemannian manifolds. It this case we obtain the vanishing of the O'Neill tensor fields $A$ and $T$; it turns out that the only nonzero curvature components are the intrinsic ones, $R^{\perp}$ and $R^{\mathcal{F}}$. In this particular case we get

$$
K_{-2,2}=K_{-1,1}=K_{1,-1}=K_{2,-2}=0
$$

and

$$
K_{0,0}^{h}=K_{0,0}^{0}+h^{2} K_{0,0}^{2}
$$

where

$$
K_{0,0}^{2}(\alpha \wedge \beta)=\sum_{a<b} \alpha^{a} \cdot \alpha^{b} \cdot R_{f_{a}, f_{b}}^{\perp} \alpha \otimes \beta .
$$

Also, we get $\nabla_{\mathcal{L}, 0,0}^{2}=\nabla_{\mathcal{L}, 1,-1}=\nabla_{\mathcal{L},-1,1}=\nabla_{\mathcal{T}, 1,-1}=\nabla_{\mathcal{T},-1,1}=0$ and, as a consequence, $\nabla^{h} \omega=\nabla_{\mathcal{L}, 0,0}^{0} \omega+h \nabla_{\mathcal{T}, 0,0} \omega$. Under these circumstances, considering also (25), the relation (22) becomes: 


$$
\begin{aligned}
\left\langle\Delta_{h} \omega_{h}, \omega_{h}\right\rangle= & \left.\left\|D_{0} \omega_{h}\right\|^{2}+h^{2} \| \nabla_{\mathcal{T}, 0,0} \omega_{h}\right) \|^{2}+h^{2}\left\langle K_{0,0}^{2} \omega_{h}, \omega_{h}\right\rangle \\
& +o\left(h^{2}\right) .
\end{aligned}
$$

Now, applying the Bochner technique for the transverse part of $\omega$, and arguing as in the classical case (see e.g. [Pet]) we obtain the following vanishing result:

Proposition 5 In the special case of a Riemannian foliation that can be locally identified with a product of two Riemannian manifolds, if the transversal curvature operator $\rho^{\perp}$ is strictly positive, then $E_{2}^{u, v}=0$ for $0<u<q$.

As an application, we might consider the case of a compact foliation ([Mo], Appendix E). Let us take a compact orientable surface $B$ of genus 2 and the Lie group $\mathrm{SO}(3, \mathbf{R})$, which is known to be a compact Lie group with strictly positive curvature [Wal]. If we take the product manifold $\widetilde{M}:=\widetilde{B} \times$ $\mathrm{SO}(3, \mathbf{R})$, where $\widetilde{B}$ is the universal covering of $B$, and consider an arbitrary homomorphism $h: \pi_{1}\left(B, x_{0}\right) \rightarrow \mathrm{SO}(3, \mathbf{R})$, with $x_{0} \in B$, then we can define the smooth diagonal action of $\pi_{1}\left(B, x_{0}\right)$ on $\widetilde{M}$ by setting

$$
R_{[\gamma]}(\widehat{x}, y)=\left(\widehat{x}[\gamma], h\left([\gamma]^{-1}\right)(y)\right)
$$

for each $[\gamma] \in \pi_{1}\left(B, x_{0}\right)$.

If we take now the quotient manifold $M=\widetilde{M} / R$, this becomes a $\operatorname{SO}(3, \mathbf{R})$ foliation. Then the terms $E_{2}^{u, v}$ of the spectral sequence vanish for $0<u<3$.

Remark 7 The above result is in fact a generalization of a result of Ph. Tondeur, M. Min-Oo and E. Ruh (see [MiRuTo]) in the special case of a compact foliation.

\section{References}

[Al] Álvarez López, J.A.: The basic component of the mean curvature of Riemannian foliations. Ann. of Global Anal. and Geom. 10, 179-194 (1992). 
[AlKo1] Álvarez López, J.A., Kordyukov, Y.A.: Long time behaviour of leafwise heat flow for Riemannian foliations. Compositio Mathematica 125, 129-153 (2001).

[AlKo2] Álvarez López, J.A., Kordyukov, Y.A.: Adiabatic limits and spectral sequences for Riemannian foliations. Geom. and Funct. Anal. 10, 977-1027 (2000).

[AlTo] Álvarez López, J.A., Tondeur, Ph.: Hodge decomposition along the leaves of a Riemannian foliation, J. of Functional Analysis 99, 443-458 (1991).

[Car] Carrière, Y.: Flots riemanniens, in "Structures transverses des feuilletages". Astérisque, 116, 31-52 (1984).

[CrPuRa] Craioveanu, M., Puta, M., Rassias, Th.M.: Old and New Aspects in Spectral Geometry. Kluwer Academic Publishers, Mathematics and Its Applications, Vol. 534, Dordrecht (2001).

[Do] Domínguez, D.: Finiteness and tenseness theorems for Riemannian foliations. Amer. J. of Math. 120, 1237-1276 (1998).

[ON] O'Neill, B.: The fundamental equations of a submersion. Michigan Math. J, 13, 459-469 (1966).

[MazMe] Mazzeo, R.R., Melrose, R.B.: Adiabatic limit, Hodge cohomology and Leray spectral sequence for a fibration. J. Diff. Geom. 31 , 185-213 (1990).

[MiRuTo] Min-Oo, M., Ruh, E.,Tondeur, P.: Vanishing theorems for the basic cohomology of Riemannian foliations. J. Reine Angew. Math. 415, 167-174 (1991).

[Mo] Molino, P.: Riemannian Foliations. Progress in Math. 73, Birkhauser Verlag, Boston Inc., Boston, MA (1988).

[MoSe] Molino, P., Sergiescu, V.: Deux remarques sur les flots riemanniens. Manuscripta Math. 51, 145-161 (1985).

[McC] McCleary, J.: User Guide to spectral Sequences, Mathematics Lectures Series. Publish or Perish Inc., Wilmington, Del. (1985). 
[Pet] Petersen, P.: Riemannian Geometry. Springer-Verlag, New York (1998).

[Re] Reinhart, B.: Foliated manifolds with bundle-like metrics. Ann. of Math. 69, 119-132 (1959).

[Rov] Rovenskii, V.: Foliations on Riemannian Manifolds and Submanifolds. Birkhäuser Verlag, Boston, MA (1998).

[Sl] Slesar, V.: The study of some problems of the geometry of Riemannian foliations. Doctoral Thesis, Universitatea de Vest din Timişoara, România, 2004.

[W] Witten, E.: Global gravitational anomalies. Comm. Math. Phys. 100, 197-229 (1985).

[Wal] Wallach, N.: Compact homogeneous Riemannian manifolds with strictly positive curvature. Ann. of Math. 96, 277-295 (1972).

VLADIMIR SLESAR

Faculty of Mathematics and Computer Sciences,

University of Craiova,

13, Al.I. Cuza st., 200585, Craiova, Romania

vlslesar@central.ucv.ro 